\begin{tabular}{|} 
Ambiente \& Água - An Interdisciplinary Journal of Applied Science \\
ISSN 1980-993X - doi:10.4136/1980-993X \\
www.ambi-agua.net \\
E-mail: ambi-agua@agro.unitau.br
\end{tabular}

\title{
Bioindicador Ceriodaphnia dubia aplicado na avaliação ecotoxicológica da água da bacia hidrográfica do rio Una
}

\author{
doi: 10.4136/ambi-agua.1371 \\ Received: 16 Aug. 2013; Accepted: 22 Nov. 2013 \\ Tatiane Alves ${ }^{1 *}$; Valter José Cobo ${ }^{1}$ \\ ${ }^{1}$ Universidade de Taubaté (UNITAU), SP \\ Programa de Pós-graduação em Ciências Ambientais \\ *Autor correspondente: e-mail: tatianealves_2004@yahoo.com.br, \\ vjcobo@gmail.com
}

\section{RESUMO}

A bacia do Una está localizada em sua maior parte no município de Taubaté, SP, contribuindo para o abastecimento de água do município. $\mathrm{O}$ objetivo da presente investigação foi caracterizar a qualidade da água do rio Una por meio do microcrustáceo Ceriodaphnia dubia como bioindicador para ensaios de toxicidade aguda e crônica. Foram coletadas duas amostras mensais de águas superficiais em cada um dos pontos amostrais, no período de março a outubro de 2011, em seis pontos distintos da bacia do Una . Foram mensurados pH, condutividade elétrica, dureza, oxigênio dissolvido e precipitação que foram correlacionados com a taxa de natalidade do organismo-teste. Não foram observadas correlações significativas para a condutividade e a precipitação em nenhuma das amostras, enquanto na sub-bacia Sete Voltas e Antas verificou-se correlação negativa para a dureza, e, para a sub-bacia Rocinha, correlação negativa para o $\mathrm{pH}$. Para o oxigênio dissolvido registrou-se correlação positiva nas sub-bacias Sete Voltas e Médio Una. Não foi detectada toxicidade aguda em nenhuma das amostras, e para todos os pontos amostrais foi verificada toxicidade crônica em algumas das análises. As análises sugerem boa qualidade das águas da sub-bacia Itaim, que apresentou toxicidade em apenas uma das análises. Para as sub-bacias Rocinha, Sete Voltas, Antas, Médio e Baixo Una foi verificada toxicidade crônica em várias amostras. O organismo-teste C. dubia mostrou eficiência como bioindicador para qualidade da água, fornecendo resultados confiáveis durante e execução dos testes.

Palavras-chave: toxicidade, cladócero, bacia do rio Una.

\section{Ecotoxicological evaluation of water of the hydrographic Basin of the Una River using the bioindicator Ceriodaphnia dubia}

\section{ABSTRACT}

The majority of the Una River Basin is located in Taubate County and contributes significantly to its water supply. The main goal of this research was to evaluate the water quality of the Una River using the microcrustacean C. dubia as bioindicator for tests of chronic and acute toxicity. Bimonthly water samples were obtained from each of six localities throughout the Una Basin, from March to October, 2011. Physical-chemical water parameters such as $\mathrm{pH}$, electrical conductivity, hardness, dissolved oxygen and precipitation were measured and correlated to the $C$. dubia reproductive rates. No significant relationships were found between the water's electrical conductivity and precipitation with respect to 
bioindicator reproductive rates. However, at the Sete Voltas, Antas and Rocinha Sub-Basins, significant interactions were detected between some water parameters and reproductive rates, suggesting that water may constrain the reproduction of $C$. dubia. Acute toxicity was not detected in any of the six sites, while chronic toxicity was recorded at Rocinha, Sete Voltas, Antas, Médio and Baixo Una Sub-Basins. In general, the water quality of the Una Basin, as indicated by the absence of acute toxicity, still remains in an acceptable conservation condition. Caution is needed, however, since slight pollution sources are causing chronic toxicity in some localities. In addition, as the microcrustacean $C$. dubia, appeared to be a reliable bioindicator in this investigation, we suggest that it be used for continuous water quality monitoring programs.

Keywords: toxicity, cladoceran, river Una basin.

\section{INTRODUÇÃO}

Os recursos hídricos da bacia hidrográfica do rio Una são utilizados para abastecimento público e industrial, e para irrigação, muito embora esta esteja diminuindo devido à substituição da agricultura pela criação de gado (Silva, 2008). Sua relevância ambiental e econômica está ligada ao fato de o rio Una ser um importante afluente do rio Paraíba do Sul e fonte de captação de pelo menos $20 \%$ de água para o abastecimento do município de Taubaté (Oliveira et al., 2006). A importância da bacia foi destacada pelo Comitê das Bacias Hidrográficas do Paraíba do Sul (CBH-PS), que estabeleceu uma ordem de priorização das bacias afluentes para ações de recuperação. A bacia do Una aparece na quarta posição de prioridade para ações de recuperação pelo referido comitê (Targa, 2009).

Sendo assim, o desenvolvimento e estabelecimento de um sistema de monitoramento ou sistema de informações sobre a qualidade da água, são necessários para efeito de tomada de decisões.

A partir de 1975, foram desenvolvidos e adaptados vários métodos de ensaios de toxicidade crônica e aguda, de curta duração, utilizando alguns grupos e espécies de organismos, entre os quais se destacam as algas unicelulares, microcrustáceos e peixes de águas continentais e marinhas (Magalhães e Ferrão-Filho, 2008),

O uso de espécies de pequeno porte e ciclo de vida não muito longo mostra-se adequado aos estudos ecotoxicológicos, e inclui-se nesse contexto o cladócero Ceriodaphnia, cujo cultivo em laboratório está bem estabelecido (Zagatto e Bertoletti, 2011). A vantagem da utilização de organismos vivos para detectar a toxicidade, baseia-se nas respostas dos organismos em relação ao meio em que vivem, pois os efeitos são realmente aqueles provocados pelas substâncias biodisponíveis (Laitano e Matias, 2006; Bastos et al., 2006).

No Brasil os ensaios ecotoxicológicos com microcrustáceos foram padronizados inicialmente com espécies exóticas, como Daphnia similis Claus, 1876 e Ceriodaphnia dubia Richard, 1894.

Os microcrustáceos Ceriodaphnia sp são mais representativos de águas continentais, no território brasileiro, quando comparados à Daphnia sp, que é utilizada em testes de toxicidade aguda. Os testes com Ceriodaphnia dubia permitem duas leituras de resultado, toxicidade aguda e crônica, sendo utilizados em avaliações da qualidade de águas superficiais e efluentes (CETESB, 2009; Rodgher e Espíndola, 2008; Portella et al., 2006).

Os testes de toxicidade com Ceriodaphnia são empregados internacionalmente pelas agências ambientais, especialmente da Comunidade Européia, Estados Unidos, Canadá, Japão e Austrália, para o controle das inúmeras descargas de efluentes líquidos industriais e municipais em ambiente aquático (Moreira et al., 2010).

Avaliações mais detalhadas acerca das características da água consideram a realização de análises químicas, as quais identificam e quantificam as concentrações das substâncias 
tóxicas, enquanto os testes de toxicidade avaliam os efeitos dessas substâncias sobre os sistemas biológicos. Assim, as análises químicas, somadas aos testes de toxicidade na bacia do rio Una, complementam-se no objetivo de caracterizar os níveis de ecotoxicidade, por meio do bioindicador Ceriodaphnia dubia, de modo a sugerir um diagnóstico de áreas prioritárias para o controle da poluição da água, nos trechos da bacia onde a qualidade da água estiver mais afetada, subsidiando políticas públicas de manejo e gestão da bacia do rio Una.

\section{MATERIAL E MÉTODOS}

\subsection{Localização da área de estudo}

A bacia hidrográfica do rio Una é formada pela união do ribeirão das Almas com o rio da Rocinha e seus afluentes, Itaim, ribeirão das Pedras ou Ipiranga, ribeirão das Sete Voltas, rio das Antas, ribeirão do Registro, e demais tributários da bacia, perfazendo uma área de 477 $\mathrm{km}^{2}$ (Figura 1) (Batista, 2005).

Foram tomadas amostras em seis localidades ao longo da bacia, denominadas sub-bacias, a saber: sub-bacia Rocinha (ponto 1 - P1) apresentando assoreamento, atividades agropastoris, mau uso do solo e a expansão de áreas urbanas; sub-bacia ribeirão das Sete Voltas (ponto 2- P2) presença de movimento de massas e atividades agropastoris; sub-bacia Antas (ponto 3 - P3) que pode ser caracterizada por eventos de assoreamento, atividades agropastoris, mau uso do solo e a expansão de áreas urbanas; sub-bacia Médio Una (ponto 4 P4), caracterizada por atividades agropastoris, industriais e expansão de áreas urbanas; sub bacia Baixo Una (ponto 5 - P5), em que se verificam atividades agropastoris e expansão de áreas urbanas e, sub- bacia Itaim (ponto 6 - P6), caracterizada por erosão, atividades agropastoris e lançamento de esgoto in natura diretamente no rio.

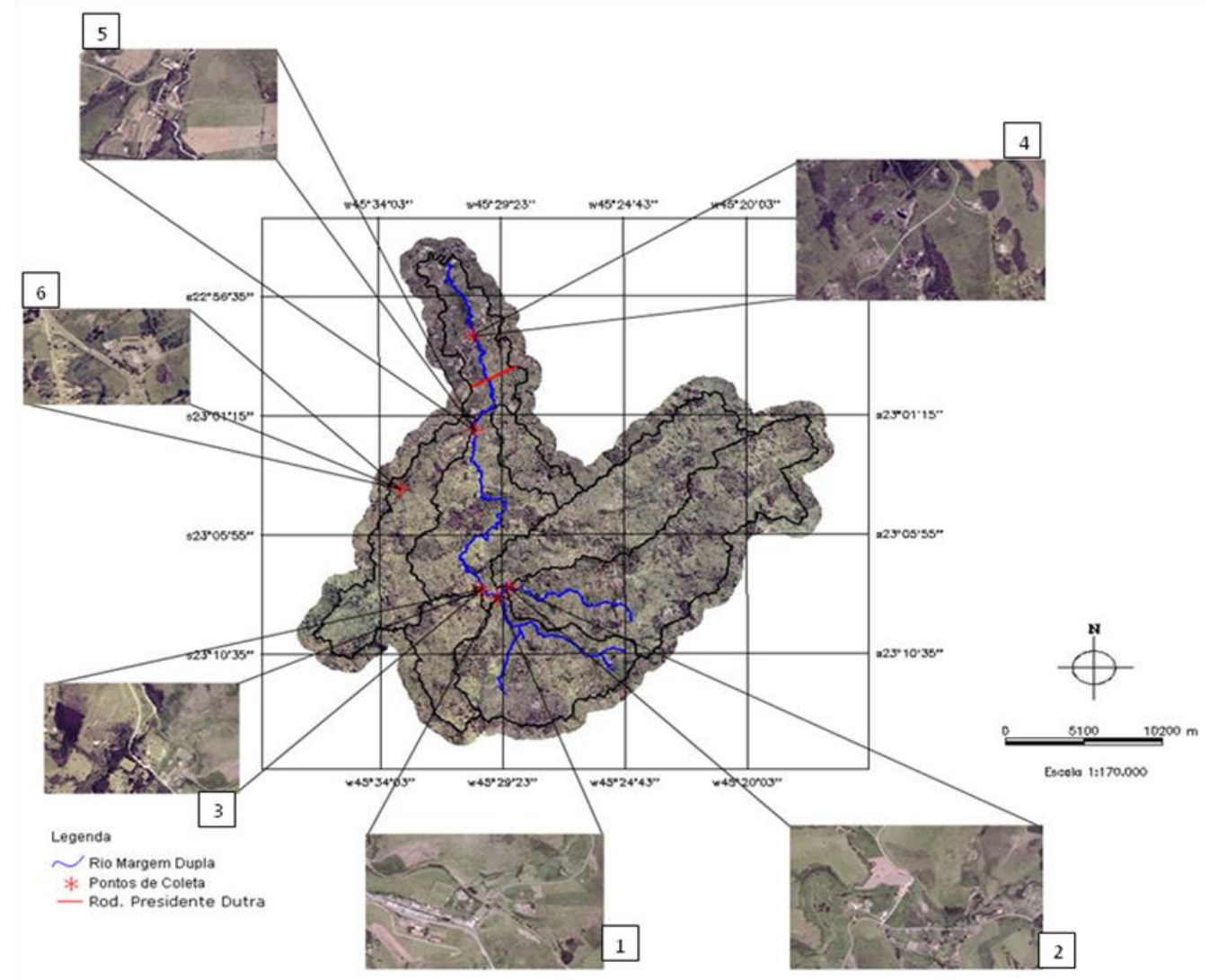

Figura 1. Localização dos pontos de coleta de água na Bacia Hidrográfica do rio Una, Taubaté, estado de São Paulo, 2011.

Fonte: Adaptado de Batista (2005). 


\subsection{Método de coleta de água}

Foram realizadas coletas bimensais de amostras de água em cada uma das localidades, de março a outubro de 2011. As amostras foram acondicionadas em frascos de polipropileno esterilizáveis e mantidas resfriadas em caixas térmicas ao abrigo de luz, para manutenção de suas características. Os ensaios laboratoriais foram iniciados em menos de três horas após cada coleta. As amostras de água utilizadas na manutenção das culturas do bioindicador foram mantidas congeladas até o momento de sua utilização também com o intuito de manter suas propriedades, sendo descongeladas em temperatura ambiente.

\subsection{Manutenção do microcrustáceo Ceriodaphnia dubia em laboratório}

Os indivíduos de $C$. dubia foram cultivados e mantidos no Setor de Ecotoxicologia do Laboratório de Processos Biológicos Aplicados ao Meio Ambiente (LPBAMA), do Programa de Pós graduação em Ciências Ambientais da Universidade de Taubaté.

As culturas foram iniciadas com indivíduos com idade entre 6 e 24 horas, individualmente, em frascos de $60 \mathrm{~mL}$ preenchidos com $15 \mathrm{~mL}$ de água reconstituída, que é caracterizada por valores entre 7,0 e 7,6 de $\mathrm{pH}$, condutividade de até $210 \mu \mathrm{c} / \mathrm{s}$, oxigênio dissolvido maior que $5 \mathrm{mg} / \mathrm{L}$ e dureza entre $40 \mathrm{mg}$ a $48 \mathrm{mg} \mathrm{CaCO} 3 / \mathrm{L}$. A água reconstituída para as análises foi captada da rede de distribuição (SABESP) do município de Taubaté. Em cada cultura havia 40 indivíduos que foram alimentados diariamente, com alga e ração, com fotoperíodo de 16 horas de luz, e mantidos em temperatura de $25^{\circ} \pm 2^{\circ} \mathrm{C}$ por um período de 14 dias, de acordo com as recomendações sugeridas por ABNT (2010), USEPA (2002) e CETESB (2004).

\subsection{Ensaio ecotoxicológico}

O ensaio foi constituído de 10 repetições para cada amostra de água de cada localidade amostral selecionada para este trabalho, cada uma com $15 \mathrm{~mL}$ de água e um indivíduo de $C$. dubia de 6 a 24 horas de idade, mantendo-se a temperatura ambiente de $25 \pm 2{ }^{\circ} \mathrm{C}$ e fotoperíodo de 16 horas de luz, alimentado diariamente com alga e ração, conforme normas da ABNT (2010), USEPA (2002) e CETESB (2004).

As amostras de água, de cada localidade amostral, foram renovadas duas vezes durante o teste, no terceiro e no quinto dia de ensaios quando foi verificada a taxa de sobrevivência das fêmeas e de natalidade do bioindicador. A água para a renovação dos ensaios foi mantida congelada até a substituição nas culturas, e submetidas ao descongelamento à temperatura ambiente. Os ensaios tiveram duração de sete dias. Após o término do teste, foi calculado o número médio de jovens produzidos por fêmea adulta e avaliado para verificação do efeito tóxico na sobrevivência e na reprodução, conforme ABNT (2010), USEPA (2002) e CETESB (2004).

O teste controle foi feito em 10 réplicas, com água de manutenção e um indivíduo de $C$. dubia, com 6 a 24 horas de idade, em cada amostra, mantendo-se rigorosamente as mesmas condições do teste toxicológico.

\subsubsection{Toxicidade aguda}

A verificação de toxicidade aguda foi acessada pela comparação da sobrevivência de $C$. dúbia, após 48 horas de ensaio com a sobrevivência do organismo do teste controle, considerando-se a contagem dos organismos imóveis e/ou mortos, para a avaliação da taxa de sobrevivência, na qual se considera toxicidade aguda quando a sobrevivência apresenta taxas menores que $50 \%$ em relação ao grupo controle, de acordo com o sugerido pela ABNT (2010). 


\subsubsection{Toxicidade crônica}

A toxicidade crônica foi estimada pela avaliação da taxa de natalidade de C. dubia durante sete dias de ensaio. Durante o teste, a cada 48 horas, as amostras de água das localidades selecionadas foram renovadas, ocasião em que foi feita a contagem dos neonatos. A toxicidade foi expressa como crônica quando houve diferença menor que $79 \%$ entre as taxas de natalidade do ensaio e do controle (Zagatto e Bertoletti, 2011).

\subsection{Análises das variáveis físico-químicas da água coletada}

Os parâmetros físico-químicos como dureza, $\mathrm{pH}$, condutividade elétrica, pluviosidade e oxigênio dissolvido foram mensurados para verificar se esses não influenciaram no comportamento dos organismos-teste interferindo nos resultados de toxicidade, pois conforme ABNT 13373 (2010) existe um limite para esses parâmetros que são ideais para a sobrevivência dos organismos-teste.

A relação de causa e efeito entre as variáveis físico-químicas mensuradas e a taxa de natalidade do bioindicador foi estimada por meio de correlação de Spearman ( $\alpha=5 \%$ ) (ZAR, 1999).

\section{RESULTADOS}

\subsection{Toxicologia Aguda}

Durante o período analisado não foi detectada toxicidade aguda para as amostras de água dos pontos de coleta, sendo registrada mortalidade inferior a $50 \%$ dos bioindicadores nos testes com água dos seis pontos de coleta (Figura 2).

\subsection{Toxicidade Crônica}

A toxicidade crônica foi registrada nos testes com água nos seis pontos de coleta de água, como mostra a Figura 3.
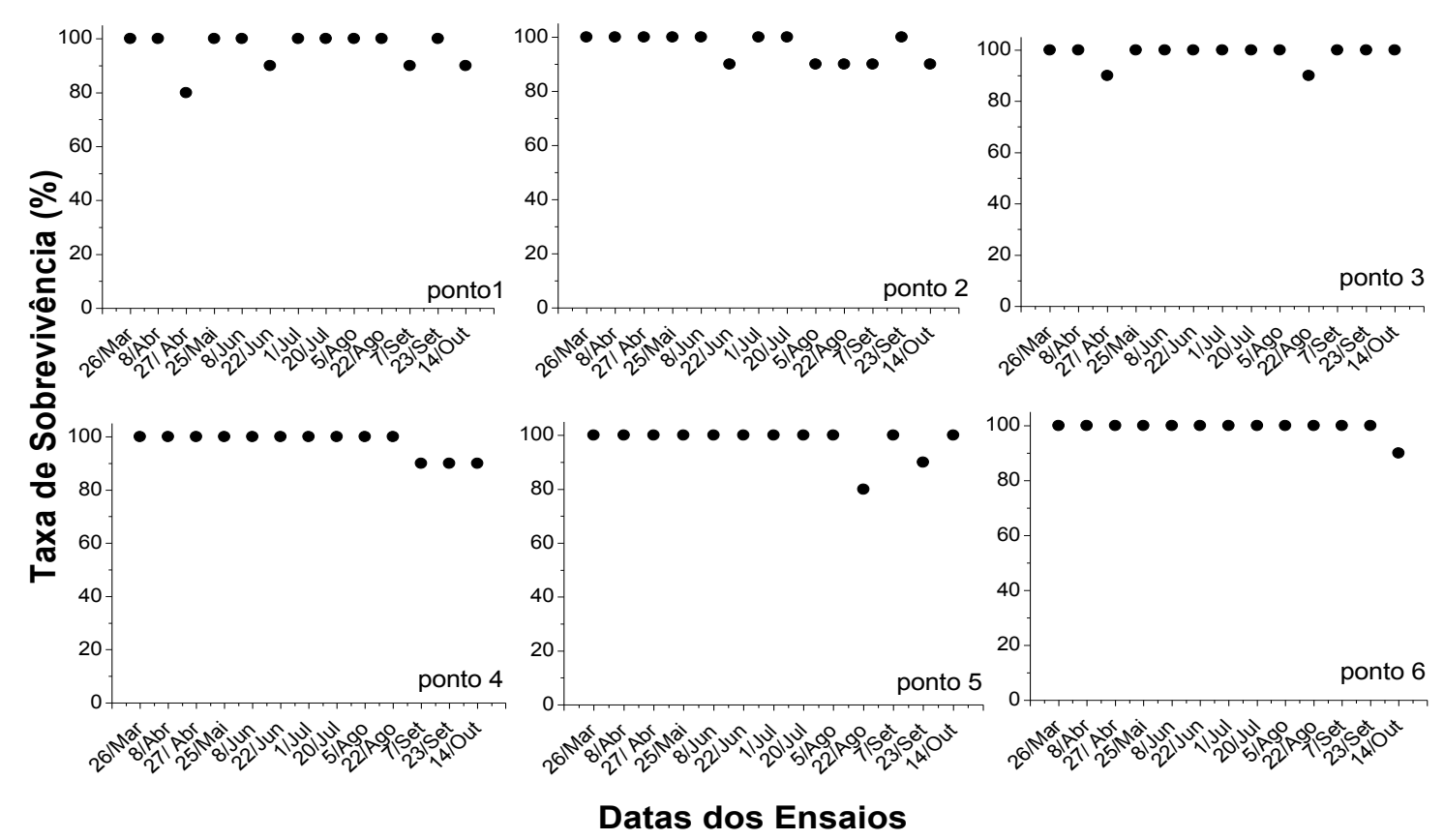

Figura 2. Taxa de sobrevivência da Ceriodaphnia dubia no período de março a outubro de 2011 na água de cada um dos seis pontos de coleta de água do rio Una, Taubaté, SP. 


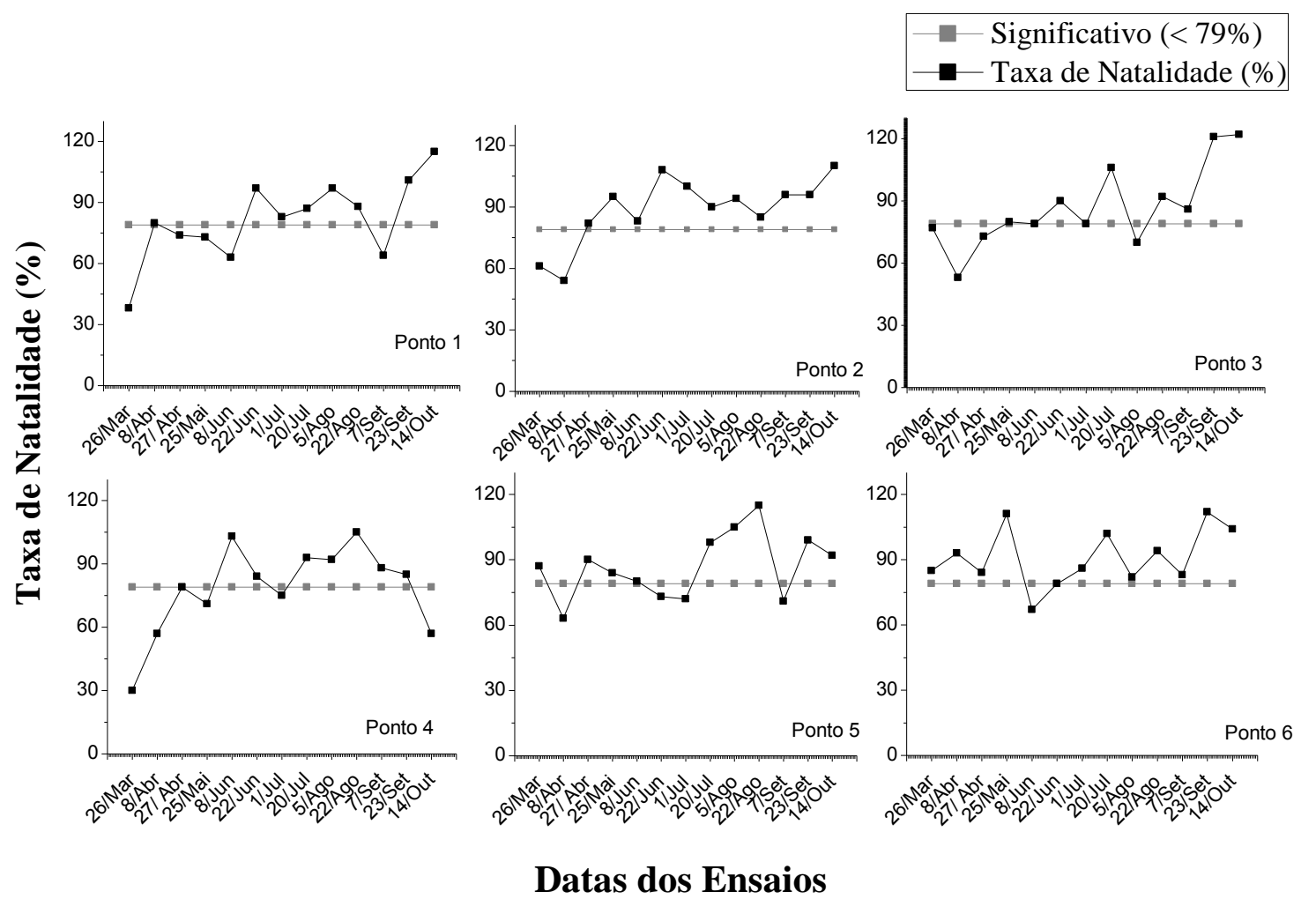

Figura 3. Taxa da natalidade da Ceriodaphnia dubia no período de março a outubro de 2011 na água de cada um dos seis pontos de coleta de água do rio Una, Taubaté, SP.

\subsection{Análises Físico-química da água}

\subsubsection{Dureza}

No ponto 1 foi verificada a maior dureza da água que foi de $67,3 \mathrm{mgCaCO}_{3 /} \mathrm{L}$ e no ponto 6 a menor dureza da água que foi de $27 \mathrm{mgCaCO}_{3} / \mathrm{L}$ (Tabela 1). Segundo a norma da ABNT 13373 (2010) a dureza ideal para o microcrustáceo varia entre $40 \mathrm{mg}$ e $47 \mathrm{mgCaCO}_{3} / \mathrm{L}$. No entanto, durante o período amostral apenas os pontos 2 e 3 apresentaram correlações negativas com a natalidade de $C$. dúbia (Tabela 2).

Tabela 1. Valores de dureza em todos os pontos amostrais, média e desvio padrão.

\begin{tabular}{|c|c|c|c|c|c|c|c|c|c|c|c|c|c|c|}
\hline \multirow[b]{2}{*}{ Amostras } & \multicolumn{12}{|c|}{ Dureza } & \multirow[b]{2}{*}{ 14/out } & \multirow[b]{2}{*}{$\begin{array}{c}\text { Média } \pm \text { desv } \\
\text { pad }\end{array}$} \\
\hline & 26/mar & 16/abr & 27/abr & 25/mai & 08/jun & 22/jun & 01/jul & 20/jul & 05/ago & 22/ago & 07/set & $22 /$ set & & \\
\hline $\mathrm{P} 1$ & 63.8 & 55.2 & 51.3 & 39.2 & 46.25 & 60.45 & 46.25 & 67.26 & 46.62 & 51.66 & 44.28 & 45.78 & 43.02 & $50.85 \pm 2.36$ \\
\hline $\mathrm{P} 2$ & 47.56 & 52.9 & 43.32 & 58.24 & 47.5 & 49.95 & 47.5 & 45.6 & 34.41 & 44.28 & 41.04 & 41.42 & 43.2 & $45.92 \pm 1.63$ \\
\hline P3 & 41.76 & 63.2 & 41.01 & 38.08 & 37.5 & 44.55 & 37.5 & 38.76 & 32.19 & 36.9 & 31.32 & 32.7 & 32.4 & $39.07 \pm 2.30$ \\
\hline $\mathrm{P} 4$ & 46.4 & 51.75 & 35.34 & 44.04 & 40 & 47.25 & 40 & 45.6 & 35.52 & 43.05 & 36.72 & 43.7 & 37.8 & $42.09 \pm 1.39$ \\
\hline P5 & 46.4 & 65.55 & 43.32 & 49.28 & 41.25 & 44.55 & 41.25 & 41.04 & 42.18 & 36.9 & 35.64 & 33.49 & 43.2 & $43.39 \pm 2.20$ \\
\hline P6 & & 33.35 & 31.92 & 35.84 & 30 & 40.5 & 30 & 31.92 & 31.08 & 44.28 & 27 & 28.37 & 28.1 & $32.7 \pm 1.44$ \\
\hline
\end{tabular}


Tabela 1. Valores dos coeficientes de correlação linear de Spearman efetuados entre a dureza e a taxa de natalidade do organismo-teste Ceriodaphnia dubia.

\begin{tabular}{cccc}
\hline \multicolumn{4}{c}{ Dureza } \\
\hline Pontos de & \multicolumn{3}{c}{ Correlação de Spearman } \\
\cline { 2 - 4 } Coleta & Coef. de Spearman & $\mathbf{t}$ & $\mathbf{p}$ \\
\hline p1 & -0.0853 & -0.2839 & 0.7818 \\
p2 & -0.6212 & -2.6292 & $0.0234^{*}$ \\
p3 & -0.5934 & -2.4451 & $0.0325^{*}$ \\
p4 & -0.2300 & -0.7839 & 0.4496 \\
p5 & -0.0496 & -0.1647 & 0.8722 \\
p6 & -0.1404 & -0.4483 & 0.6635 \\
\hline
\end{tabular}

\subsubsection{Potencial Hidrogeniônico (pH)}

Os valores do $\mathrm{pH}$ encontrados foram similares entre os seis pontos analisados (tabela 3 ), todos dentro dos limites estabelecidos pelo CONAMA 357 (Brasil, 2005), que para rios de classe dois, classificação do Una, estão entre 6 e 9. Para o organismo estudado o pH ideal que está entre 7,0 e 7,6 (ABNT, 2010).

Apenas para o ponto 1 foi verificada correlação negativa com a natalidade de $C$. dubia (Tabela 4).

Tabela 3. Valores do $\mathrm{pH}$ em todos os pontos amostrais, média e desvio padrão.

\begin{tabular}{|c|c|c|c|c|c|c|c|c|c|c|c|c|c|c|}
\hline & & & & & & & pH & & & & & & & \\
\hline Amostras & 26/mar & 08/abr & 27/abr & $25 /$ mai & 08/jun & 22/jun & 01/jul & 20/jul & 05/ago & 22/ago & 07/set & 23/set & 14/out & $\underset{\text { pad }}{\text { Média } \pm \text { desv }}$ \\
\hline P1 & 6.96 & 6.91 & 7.12 & 6.6 & 6.36 & 6.29 & 6.15 & 6.39 & 6.08 & 6.2 & 6.39 & 6.33 & 6.2 & $6.46 \pm 0.09$ \\
\hline $\mathrm{P} 2$ & 6.98 & 7.02 & 7.06 & 6.7 & 6.53 & 6.45 & 6.36 & 6.55 & 6.24 & 6.44 & 6.68 & 6.53 & 6.44 & $6.61 \pm 0.07$ \\
\hline P3 & 6.94 & 7.12 & 6.66 & 6.8 & 6.62 & 6.54 & 6.46 & 6.59 & 6.29 & 6.57 & 6.78 & 6.63 & 6.53 & $6.65 \pm 0.06$ \\
\hline $\mathrm{P} 4$ & 6.86 & 7.06 & 6.43 & 6.8 & 6.6 & 6.56 & 6.49 & 6.67 & 6.38 & 6.65 & 6.83 & 6.69 & 6.54 & $6.66 \pm 0.05$ \\
\hline P5 & 6.93 & 7.15 & 7.15 & 6.8 & 6.7 & 6.69 & 6.56 & 6.72 & 6.4 & 6.71 & 6.93 & 6.77 & 6.57 & $6.77 \pm 0.06$ \\
\hline P6 & 6.9 & 7.18 & 6.87 & 6.9 & 6.74 & 6.72 & 6.63 & 6.81 & 6.49 & 6.72 & 7.05 & 6.82 & 6.65 & $6.81 \pm 0.05$ \\
\hline
\end{tabular}

Tabela 4. Valores dos coeficientes de correlação linear de Spearman efetuados entre o pHe a taxa de natalidade do organismo-teste, Ceriodaphnia dubia.

\begin{tabular}{cccc}
\hline \multicolumn{4}{c}{$\mathbf{p H}$} \\
\hline Pontos de & \multicolumn{3}{c}{ Correlação de Spearman } \\
\hline Coleta & Coef. de Spearman & $\mathbf{t}$ & $\mathbf{p}$ \\
\hline p1 & -0.5572 & -2.2258 & $0.048^{*}$ \\
p2 & -0.4207 & -1.5380 & 0.1522 \\
p3 & -0.2363 & -0.8064 & 0.4371 \\
p4 & -0.1871 & -0.6316 & 0.5405 \\
p5 & 0.0400 & 0.1328 & 0.8968 \\
p6 & 0.3526 & 1.2498 & 0.2372 \\
\hline
\end{tabular}

\subsubsection{Condutividade elétrica}

Todas as amostras apresentaram resultados dentro do limite ideal da condutividade elétrica para C. dubia entre 72 a $212 \mu \mathrm{S} / \mathrm{cm}$ (Zagatto e Bertoletti, 2011; ABNT, 2010), 
variando entre $81,1 \mu \mathrm{S} / \mathrm{cm}$ no ponto 2 e $112,9 \mu \mathrm{S} / \mathrm{cm}$ no ponto 1 (Tabela 5), para os quais não foi verificada correlação significativa com a reprodução (Tabela 6).

Tabela 5. Valores da condutividade elétrica em todos os pontos amostrais, média e desvio padrão.

\begin{tabular}{ccccccccccccccccc}
\hline \multicolumn{11}{c}{ Condutividade elétrica $(\boldsymbol{\mu}$ S/cm) } \\
\hline Amostras & 26/mar & 08/abr & 27/abr & 25/mai & 08/jun & 22/jun & 01/jul & 20/jul & 05/ago & 22/ago & 07/set & 22/set & 14/out & $\begin{array}{c}\text { Média } \pm \text { desv } \\
\text { pad }\end{array}$ \\
\hline P1 & 109.3 & 112.9 & 101.4 & 105.8 & 102 & 109 & 105 & 109.6 & 108 & 105.6 & 107.5 & 108.8 & 108.7 & $107.2 \pm 0.88$ \\
P2 & 88.8 & 96.2 & 81.7 & 81.1 & 92.9 & 84.4 & 82.1 & 88.5 & 87 & 84.9 & 93.4 & 89.5 & 97.6 & $88.32 \pm 1.52$ \\
P3 & 90.5 & 96.6 & 87 & 84.4 & 84.7 & 84.5 & 83.7 & 85 & 84.3 & 83.1 & 84.6 & 85.6 & 89.3 & $86.41 \pm 1.04$ \\
P4 & 89.4 & 93.9 & 83.4 & 84.2 & 82.8 & 85.2 & 86.6 & 87.2 & 85.3 & 89.2 & 92.2 & 94 & 94.3 & $88.28 \pm 1.16$ \\
P5 & 88.8 & 97.5 & 83.8 & 82.2 & 86.3 & 88.4 & 83.7 & 85.2 & 81.3 & 86 & 89.9 & 88.8 & 106.9 & $88.37 \pm 1.93$ \\
P6 & 81.1 & 77.9 & 79 & 81.8 & 77.9 & 85.9 & 86.1 & 81.7 & 85.8 & 86.6 & 86 & 88.1 & 93 & $83.92 \pm 1.23$ \\
\hline
\end{tabular}

Tabela 6. Valores dos coeficientes de correlação linear de Spearman efetuados entre a condutividade elétrica e a taxa de natalidade do organismo-teste Ceriodaphnia dubia.

\begin{tabular}{cccc}
\hline \multicolumn{4}{c}{ Condutividade } \\
\hline Pontos de & \multicolumn{3}{c}{ Correlação de Spearman } \\
\cline { 2 - 4 } Coleta & Coef. de Spearman & $\mathbf{t}$ & $\mathbf{P}$ \\
\hline p1 & 0.3384 & 1.1926 & 0.2580 \\
p2 & 0.3191 & 1.1168 & 0.2878 \\
p3 & 0.1758 & 0.5924 & 0.5656 \\
p4 & -0.2861 & -0.9903 & 0.3433 \\
p5 & 0.1240 & 0.4143 & 0.6866 \\
p6 & -0.1265 & -0.4231 & 0.6804 \\
\hline
\end{tabular}

\subsubsection{Oxigênio Dissolvido}

O teor de oxigênio dissolvido variou entre $5 \mathrm{mg} / \mathrm{L}$ e $13 \mathrm{mg} / \mathrm{L}$, nos pontos 4 e 6 respectivamente, portanto dentro do limite recomendado pela ABNT 13373 (2010) (tabela 7). O teor de OD estabelecido pelo CONAMA 357 (Brasil, 2005) para rios de classe dois, classificação do Una, é maior que $5 \mathrm{mg} / \mathrm{L}$. Porém, para o organismo teste, teores acima de 3 $\mathrm{mg} / \mathrm{L}$ mostram-se adequados para sua sobrevivência (ABNT, 2010). Esses valores podem explicar o fato de haver correlações positivas entre o teor de oxigênio e a natalidade de $C$. dubia para dois pontos (2 e 4) (Tabela 8).

Tabela 7. Valores do teor de oxigênio dissolvido em todos os pontos amostrais, média e desvio padrão.

\begin{tabular}{ccccccccccccc}
\hline \multicolumn{10}{c}{ Oxigênio Dissolvido } \\
\hline Amostras & 26/mar & 25/mai & 08/jun & 22/jun & 01/jul & 20/jul & 05/ago & 22/ago & 07/set & 22/set & 14/out & $\begin{array}{c}\text { Média } \pm \text { desv } \\
\text { pad }\end{array}$ \\
\hline P1 & 7.1 & 7.2 & 8.8 & 7.7 & 9.3 & 7.8 & 9.8 & 10.4 & 8.6 & 6.1 & 7.5 & $8.21 \pm 0.36$ \\
P2 & 6.9 & 6.6 & 7.9 & 6.1 & 7.3 & 8.9 & 10.8 & 9.6 & 8.5 & 6.4 & 7.3 & $7.85 \pm 0.41$ \\
P3 & 7.1 & 7.9 & 10.5 & 5.5 & 7.7 & 8.4 & 8.6 & 13.2 & 9.7 & 6 & 7.5 & $8.37 \pm 0.60$ \\
P4 & 6.4 & 7.2 & 8.3 & 5.5 & 6.3 & 8.1 & 7.7 & 9.3 & 8.4 & 5.9 & 4.7 & $7.07 \pm 0.39$ \\
P5 & 7 & 6.4 & 9.7 & 6.3 & 7.1 & 8.7 & 6.6 & 10.9 & 5.6 & 7.1 & 7.1 & $7.50 \pm 0.44$ \\
P6 & 6.6 & 6.7 & 9.4 & 6.9 & 7.9 & 7.5 & 7 & 9.9 & 5.4 & 7.1 & 6 & $7.31 \pm 0.37$ \\
\hline
\end{tabular}


Tabela 8. Valores dos coeficientes de correlação linear de Spearman efetuados entre o oxigênio dissolvido e a taxa de natalidade do organismo-teste Ceriodaphnia dubia.

\begin{tabular}{cccc}
\hline \multicolumn{4}{c}{ Oxigênio Dissolvido } \\
\hline Pontos de & \multicolumn{3}{c}{ Correlação de Spearman } \\
\cline { 2 - 4 } Coleta & Coef. De Spearman & t & P \\
\hline p1 & 0.4055 & 1.3307 & 0.2159 \\
p2 & 0.6461 & 2.5397 & $0.0317^{*}$ \\
p3 & 0.0545 & 0.1639 & 0.8734 \\
p4 & 0.697 & 2.9164 & $0.0171^{*}$ \\
p5 & 0.2294 & 0.7069 & 0.4975 \\
p6 & -0.2091 & -0.6415 & 0.5372 \\
\hline
\end{tabular}

\subsubsection{Precipitação}

A precipitação no município de Taubaté, durante o período amostral, variou de $1,2 \mathrm{~mm}$ a 206,8 mm, em julho e março respectivamente (Figura 4), não sendo detectada correlação significativa com a natalidade de $C$. dubia, para nenhum dos pontos amostrais (Tabela 9).

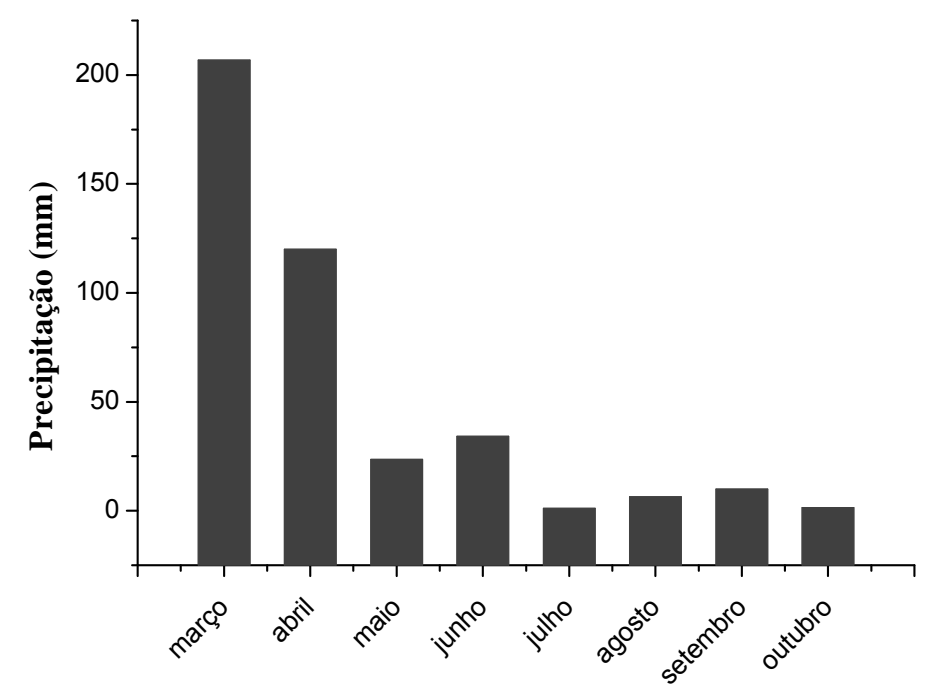

Média Mensal da Precipitação

Figura 4. Média Mensal da Precipitação no município de Taubaté, estado de São Paulo, de março a outubro de 2011. Estação de Meteorologia UNITAU, Departamento de Agronomia.

Tabela 9. Valores dos coeficientes de correlação linear de Spearman para a pluviosidade e a taxa de natalidade do organismo-teste Ceriodaphnia dubia.

\begin{tabular}{cccc}
\hline \multicolumn{4}{c}{ Pluviosidade } \\
\hline Pontos de & \multicolumn{3}{c}{ Correlação de Spearman } \\
\cline { 2 - 4 } Coleta & Coef. de Spearman & $\mathbf{t}$ & P \\
\hline p1 & 0.2121 & 0.7198 & 0.4867 \\
p2 & -0.026 & -0.0864 & 0.9327 \\
p3 & 0.2006 & 0.6793 & 0.511 \\
p4 & 0.16 & 0.5375 & 0.6016 \\
p5 & 0.0949 & 0.3161 & 0.7578 \\
p6 & -0.2712 & -0.9346 & 0.37 \\
\hline
\end{tabular}




\section{DISCUSSÃO}

$\mathrm{Na}$ presente investigação os ensaios não revelaram toxicidade aguda, considerando os índices de mortalidade inferiores a 50\% dos organismos bioindicadores para todos os pontos de coleta. Os resultados de toxicidade aguda desta investigação permitiram verificar que trechos das sub-bacias do Una, ainda não se encontram contaminados em níveis tais que possam causar efeitos deletérios agudos à biota. Corroborando com o resultado desta pesquisa Deus (2003), não constatou toxicidade aguda no Ribeirão Itaim, sub-bacia do Una, em ensaios com o mesmo bioindicador. Oliveira (2006), também não encontrou toxicidade aguda em nenhum dos pontos amostrais nas águas do Ribeirão Sete Voltas, sub-bacia do Una, no qual foram realizados testes de toxicidade com o mesmo bioindicador.

A área analisada possui atividades agropastoris e a expansão da área urbana, porém não é um local de despejos industriais e Oliveira-Filho et al. (2011), afirma que a presença de produtos químicos na água é o principal fator para a ocorrência de efeitos tóxicos em $C$. dubia e Danio rerio. Hu et al. (2012) conclui que a sinergia das substâncias químicas pode trazer um efeito tóxico para espécies aquosas, como $C$. dubia, através da captação de partículas.

Com relação a toxicidade crônica, quando a reprodução é inferior a $79 \%$ em relação ao controle (100\%), foi detectada em todos os pontos amostrados, em pelo menos uma das análises, indicando que o agente tóxico foi liberado em eventos periódicos e em concentrações subletais, durante um prolongado período, esporádicos ou não.

As sub-bacias Rocinha, Ribeirão Sete Voltas, Antas, Médio e Baixo Una, são circundadas por propriedades agrícolas e de pastagem, o que sugere o efeito de defensivos agrícolas e veterinários, os quais podem, ao longo do tempo, ter influenciado na toxicidade encontrada, em alguns dos ensaios, nestas áreas. O mesmo foi descrito por Barbosa (2010), que identificou toxicidade em nascentes da bacia do rio Mogi-Guaçu, município de Bom Repouso, sul de Minas Gerais, e descreve que dois pontos, dos três avaliados, para os quais foram registradas toxicidade crônica, estão próximos a áreas agrícolas e pastagem.

$\mathrm{O}$ rio Rocinha, um dos formadores o rio Una, foi o que mais apresentou toxicidade crônica, mesmo não observando ao longo da bacia um gradiente decrescente de toxicidade, no sentido da nascente à foz, é possível que os poluentes descartados próximos a nascente, possam, ao longo do rio, ser retidos por sólidos em suspensão presentes na água. Contribuindo com o exposto, Rodgher et al. (2005), concluíram que os descartes de poluentes próximo a nascente do rio Tietê, por serem retidos por sólidos em suspensão, diminuíram a toxicidade ao longo do corpo hídrico.

Os resultados obtidos para o rio Itaim foi a constatação da toxicidade crônica em apenas uma das análises, e nas proximidades desse rio se encontra despejo de esgoto doméstico. $\mathrm{O}$ que pode caracterizar um aumento na fecundidade dos cladóceros pela disponibilidade de alimento na água. Resultado esse comprovado por Takenaka et al. (2006) que analisaram as águas dos reservatórios de Anhumas e Cruzes, no município de Araraquara, e observaram que as amostras de água além de não causarem toxicidade ao organismo-teste promoveram um aumento na taxa de natalidade e, atribuíram esse fato a maior disponibilidade de alimento no corpo hídrico. Oliveira-filho et al. (2011) também atribuiu a ausência de toxicidade, na maioria dos ensaios, com o microcrustáceo C. dubia, ao efluente doméstico, pois os efluentes são extremamente variáveis em sua composição e afirma que o monitoramento completo deve continuar, em relação as constantes mudanças em geral encontrado na composição de esgoto.

Durante o período amostral as sub-bacias Ribeirão Sete Voltas e Antas apresentaram correlação negativa entre a dureza e a natalidade de $C$. dúbia, sendo que para os demais pontos amostrais a dureza parece não ter interferido no processo reprodutivo. Parâmetro importante a ser analisado, pois de acordo com Magalhães (2003), a dureza é influenciada 
prioritariamente por cálcio e magnésio, e a ocorrência excessiva do cálcio retira o oxigênio da água, podendo ter efeito letal para o organismo-teste.

Os resultados de $\mathrm{pH}$ indicam que os rios não sofreram impactos com substâncias ácidas ou básicas capazes de alterar de modo significativo o rio analisado no período estudado. De acordo com Tavares e Rocha (2001), águas ligeiramente ácidas a alcalinas são ideais para o desenvolvimento e reprodução dos cladóceros, o que esclarece os resultados desta pesquisa.

O parâmetro da condutividade elétrica não influenciou de maneira significativa o organismo teste, e segundo Saraiva et al. (2009), valores de condutividade acima do limite estabelecido pela ABNT 13373 (2010) indicam ambientes impactados, o que pode esclarecer o fato deste parâmetro não ter influenciado nos testes do presente estudo. Os mesmos resultados foram obtidos por Santos (2009) e Rodrigues (2009) também em pontos amostrais da bacia hidrográfica do rio Una.

Os valores de oxigênio dissolvido obtiveram correlação positiva com a natalidade de $C$. dubia nas sub-bacias Ribeirão Sete Voltas e Médio Una, e em todas as análises o teor de oxigênio dissolvido esteve dentro das normas estabelecidas pela ABNT (2010), favorecendo a sobrevivência e natalidade do organismo teste. O Relatório da CETESB (2009) afirma que águas limpas ocorrem maiores concentrações de oxigênio dissolvido na água, o que comprova os achados nesta pesquisa.

Não foi observada correlação significativa entre a pluviosidade e a reprodução, e este fato pode ter ocorrido devido ao baixo índice pluviométrico no período das amostras, que foram coletadas no outono, inverno e primavera. Segundo Folhes e Fisch (2006), para a região de Taubaté, se observam redução da precipitação em abril (outono), enquanto os meses de inverno apresentam um total de chuvas muito pequeno, e o aumento da precipitação só se inicia em novembro (primavera). Silva (2008) afirma que o baixo índice pluviométrico também contribuiu para a redução da taxa de coliformes totais na bacia hidrográfica do Una.

Carvalho et al. (2000) avaliaram a água de dois ribeirões, do Onça e do Feijão, em São Carlos - SP, e observaram que a correlação da pluviosidade com alguns parâmetros físicoquímicos, não foi significativa para as coletas realizadas no inverno, e significativas quando realizadas no verão, os autores caracterizam a água desses dois ribeirões como "melhor" no inverno do que no verão, e atribuem este efeito à precipitação, que ao contrário de diluir a concentração de resíduos, aumentaria o escoamento superficial do solo, resultando em um Índice de Qualidade de Água (IQA) menor e diminuindo a qualidade da água, o que corrobora com os resultados encontrados na presente pesquisa.

Botelho et al. (2012) que analisaram o córrego Piracicamirim, a montante e a jusante de uma indústria de açúcar e etanol através de testes de toxicidade, e afirmaram que não houve efeito tóxico da água para D. magna, C. dubia e C. silvestrii, mostrando que as três espécies apresentaram sensibilidades semelhantes em relação à água dos pontos de amostragem. Muitos autores declaram a eficiência deste microcrustáceo também para detectar a toxicidade em ambientes com despejos de indústrias químicas (e.g.: Gómez et al., 2008; Arimoto, 2009; Radic et al., 2010).

O microcrustáceo $C$. dubia se mostrou um organismo sensível a possíveis impactos ambientais, demonstrando-se um bioindicador confiável para os ensaios ecotoxicológicos o que corrobora com diversos autores (e.g.: Portela, 2002; Portela et al., 2006; ABNT, 2010; CETESB, 2009).

\section{CONCLUSÃO}

Durante o período amostrado a bacia do Rio Una não apresentou sinais de toxicidade aguda em nenhuma das localidades amostradas. Por outro lado, registros de toxicidade aguda foram feitos em todas as sub bacias avaliadas, em pelo menos uma das amostras, o que sugere 
a necessidade de programas de gerenciamento ambiental que possam detectar com antecedência a ocorrência de situações mais sérias de poluição da água.

O organismo-teste $C$. dubia mostrou eficácia com bioindicador para qualidade da água, fornecendo resultados confiáveis durante e execução dos testes desta investigação, o que permite sugerir a sua utilização em programas de monitoramento ambiental em sistemas aquáticos dulcícolas.

\section{REFERÊNCIAS}

ASSOCIAÇÃO BRASILEIRA DE NORMAS TÉCNICAS - ABNT. NBR 13373. Ecotoxicologia aquática- Toxicidade crônica- Métodos de ensaio com Ceriodaphnia spp. (Crustacea, Cladócera). Rio de Janeiro, 2010.

ARIMOTO, F. O. Impact of rubber effluent discharges on the water quality and macroinvertebrate community assemblages in a forest stream in the Niger Delta. $\begin{array}{lllllll}\text { Chemosphere, } & \text { v. } & 77, \quad \text { n. } & 3, & \text { p. } & 440-449,\end{array}$ http://dx.doi.org/10.1016/j.chemosphere.2009.06.031

BARBOSA, D. S. Avaliação e Identificação de Toxicidade (Fase I) em nascentes da bacia do rio Mogi-Guaçu município de Bom Repouso - MG. 2010. 105f. Dissertação (Mestrado) - Escola de Engenharia de São Carlos, Universidade de São Paulo, São Carlos, 2010.

BASTOS, I. C. O.; LOVO, I. V.; ESTANISLAU, C. A. M.; SCOSS, L. M. Utilização de bioindicadores em diferentes hidrossistemas de uma indústria de papeis reciclados em Governador Valadares-MG. Engenharia Sanitária e Ambiental, v. 11, n 3, p. 203 211, 2006. http://dx.doi.org/10.1590/S1413-41522006000300003

BATISTA, G. T. Base de dados ambientais na Bacia do Rio Una. 2005. Disponível em: http://www.agro.unitau.br/una/una_bacia_rio.html. Acesso em: 15 abr. 2010.

BOTELHO, R. G.; MACHADO NETO, L.; OLINDA, R. A.; DIAS, C. T.; TORNISIELO, V. L. Water quality assessment in piracicamirim creek upstream and downstream a sugar and ethanol industry through toxicity tests with cladocerans. Brazilian Archives of Biology and Technology, v. 55, n. 4, p. 631-636, 2012. http://dx.doi.org/10.1590/S151689132012000400020

BRASIL. Conselho Nacional do Meio Ambiente - CONAMA. Resolução n³57 de 17 de março de 2005. Disponível em: http://www.mma.gov.br/port/conama/res/res05/res35705.pdf. Acesso em: 13 jan. 2011.

CARVAlHO, A. R.; SCHLITTLER, F. H. M.; TORNISIELO, V. L. Relações da Atividade Agropecuária com Parâmetros Físicos Químicos da Água. Química Nova, v. 23, n. 5, p. 618-622, 2000. http://dx.doi.org/10.1590/S0100-40422000000500009

COMPANHIA AMBIENTAL DO ESTADO DE SÃo PAUlO - CETESB. Métodos de avaliação de poluentes a organismos aquáticos. São Paulo, 2004. (Cursos e Treinamentos).

COMPANHIA AMBIENTAL DO ESTADO DE SÃO PAULO - CETESB. Significado ambiental e sanitário das variáveis de qualidade das águas e dos sedimentos e metodologias analíticas e de amostragem. São Paulo, 2009. (Série Relatórios). 
DEUS, L. B. F. Avaliação da toxicidade no ribeirão Itaim, Taubaté-SP, por meio do bioindicador Ceriodaphnia dubia Richard 1894 (Crustácea-Cladocera). 2003. Trabalho de Conclusão do Curso (Graduação em Biologia) - Universidade de Taubaté, Taubaté, 2003.

FOLHES, M. T.; FISCH, G. Caracterização climática e estudo de tendências nas séries temporais de temperatura do ar e precipitação em Taubaté (SP). Revista Ambi-Água, Taubaté, v. 1, n.1, p. 61-71, 2006. http://dx.doi.org/10.4136/ambi-agua.6

GÓMEZ, N.; SIERRA, M. V.; CORTELEZZI, A.; RODRIGUES CAPÍTULO, A. Effects of discharges from the textile industry on the biotic integrity of benthic assemblages. Ecotoxicology and Environmental Safety, v. 69, n. 3, p. 472-479, 2008. http://dx.doi.org/10.1016/j.ecoenv.2007.03.007

HU, J.; WANG, D.; WANG, J.; WANG, J. Toxicity of lead on Ceriodaphnia dubia in the presence of nano-CeO2 and nano-TiO2. Chemosphere, v. 89, p. 536-541, 2012. http://dx.doi.org/10.1016/j.chemosphere.2012.05.045

LAITANO, K. S.; MATIAS, W. G. Testes de toxicidade com Daphnia magna: Uma ferramenta para avaliação de um reator Experimental UASB. Journal of the Brazilian Society of Ecotoxicology, v. 1, n. 1, p. 43-47, 2006.

MAGAlHÃES, N. P. Teste de toxicidade com organismos aquáticos, Daphnia similis: uma ferramenta para avaliação do impacto ambiental, dos afluentes nas águas do rio Paraíba do Sul. 2003. Dissertação (Mestrado em Ciências Ambientais) - Universidade de Taubaté, Taubaté, 2003.

MAGALHÃES, D. P.; FERRÃO-FILHO, A. S. A ecotoxicologia como ferramenta no biomonitoramento de ecossistemas aquáticos. Oecologia Brasiliensis, v. 12, n. 3, p. 355-381, 2008.

MOREIRA, L. E. B.; LOMBARDI, J. V.; MERCANTE, C. T. J.; BAZANTEYAMAGUISHI, R. Análise ecotoxicológica em viveiro de carcinicultura de água doce, utilizando o cladócero Ceriodaphnia dubia como organismo-teste. Boletim do Instituto de Pesca, v. 36, n.1, p. 25-38, 2010.

OLIVEIRA, E. S.; BATISTA, G. T.; DIAS, N. W. Análise Físico-Ambiental da Bacia do Una: Suporte de Análise Físico-Químico da Água- SP. In: SEMINÁRIO DE SENSORIAMENTO REMOTO DE GEOPROCESSAMENTO DO VALE DO PARAÍBA, 1., 7 de dez. 2006. Anais... Taubaté, 2006.

OLIVEIRA-FILHO, E. C.; RAMOS, M. G.; FREIRE, I. S.; MUNIZ, D. H. F. Comparison between the efficiency of two bioindicators for determining surface water quality in an urban environment. Acta Scientiarum. Biological Sciences, v. 33, n. 3, p. 311-317, 2011.

OliveIRA, L. H. Avaliação da Toxicidade Aguda com organismo Aquático Daphnia Similis e Ceriodaphnia Dubia (Crustácea, Cladocera), em águas dos Afluentes do Ribeirão das Sete Voltas, Bacia Hidrográfica do Rio Una No Município de Taubaté - SP. 2006. 57 f. Monografia (Graduação) - Faculdades Integradas Teresa D’Ávila, Lorena, 2006. 
PORTELA, C. M. S. Avaliação ecotoxicológica do Arroio Sapucaia - RS, com a utilização de diferentes metodologias em água superficial e sedimento. 2009. 105f. Dissertação (Mestrado em Ecologia) - Universidade Federal do Rio Grande do Sul, Porto Alegre, 2002.

PORTELA, C. M. S.; ARENZON, A.; RAYA-RODRIGUES, M. T. Avaliação ecotoxicológica do sedimento do Arroio Sapucaia - RS, Brasil, através de ensaios de toxicidade utilizando diferentes amostradores e organismos-teste. Journal of the Brazilian Society of Ecotoxicology, v. 1, n. 2, p. 119-122, 2006.

RADIC, S.; STIPANICEV, D.; CVJETKO, P.; MIKELIC'I, L.; RAJCIC'M, M.; SIRAC, S. et al. Ecotoxicological assessment of industrial effluent using duckweed (Lemna minor L.) as a test organism. Ecotoxicology, v. 19, n. 1, p. 216-222, 2010. http://dx.doi.org/10.1007/s10646-009-0408-0

RODGHER, S.; ESPÍNDOLA, E. L. G. The influence of algal densities on the toxicity of chromium for Ceriodaphnia dubia Richard (Cladocera, Crustacea). Brazilian Journal of Biology, v. 68, n. 2, p. 341-348, 2008. http://dx.doi.org/10.1590/S1519-69842008000200015

RODGHER, S.; ESPÍNDOLA, E. L. G.; ROCHA, O.; FRACÁCIO, R.; PEREIRA, R. H. G.; RODRIGUES, M. H. S. Limnological and ecotoxicological studies in the cascade of reservoirs in the Tietê river (São Paulo, Brasil). Brazilian Journal of Biology, v. 65, n. 4, p. 697-710, 2005. http://dx.doi.org/10.1590/S1519-69842005000400017

RODRIGUES, C. R. F. Avaliação do grau de toxicidade das águas superficiais e bruta captada no rio Paraíba do Sul no trecho do município de Jacareí-SP, utilizando como ferramenta o microcrustáceo Ceriodaphnia dubia. 2009. 25f. Monografia (Graduação) - Universidade de Taubaté, Taubaté, 2009.

SANTOS, S. A. Aplicação do teste de toxicidade aguda, utilizando o organismo aquático Ceriodaphnia Richar, 1984 (Crustacea-Cladocera) na água do Ribeirão Galega, município de Pindamonhangaba-SP. 2009. 38f. Monografia (Graduação) Universidade de Taubaté, Taubaté, 2009.

SARAIVA, V. K.; NASCIMENTO, M. R. L.; PALMIERI, H. E.; JACOMINO, V. M. F. Avaliação da qualidade de sedimentos - estudo de caso: sub-bacia do ribeirão Espírito Santo, afluente do rio São Francisco. Química Nova, v. 32, n. 8, p. 2009. http://dx.doi.org/10.1590/S0100-40422009000800003

SILVA, A. B. A. Qualidade sanitária das águas do Rio Una, São Paulo, Brasil, no período de chuvas. Revista Biociências, Taubaté, v. 14, n. 1, 2008.

TAKENAKA, R. A.; SOTERO-SANTOS, R. B.; ROCHA, O. Water quality assessment by ecotoxicological and limnological methods in water supplies, Southeast Brasil. Ecotoxicology, v. 15, p. 73-82, 2006. http://dx.doi.org/ 10.1007/s10646-005-0048-y

TARGA, M. S. Estudo hidrológico da Bacia do Rio Una: subsídios para estabelecimento de planos de macrodrenagem no Vale do Paraíba do Sul-SP. Taubaté: Programa de PósGraduação em Ciências Ambientais, UNITAU, 2009. p. 1-14.

TAVARES, L. H. S.; ROCHA, O. Produção de plâncton (Fitoplâncton e Zooplâncton) para alimentação de organismos aquáticos. São Carlos: RIMA, 2001. p. 106.

UNITED STATES. Environmental Protection Agency - USEPA. Methods for measuring the acute toxicity of effluents and receiving waters to freshwater and marine organisms. EPA-821-R-02-012. Washington, DC., 2002. 8p. 
ZAGATTO, P. A.; BERTOLETTI, E. Ecotoxicologia aquática: princípios e aplicações. 2. ed. São Carlos, RIMA, 2011.

ZAR, J. H. Biostatistical analysis. 4. ed. Upper Saddle River: Prentice-Hall, 1999. 663 p. 